\title{
CARACTERÍSTICAS DO ATENDIMENTO DE PACIENTES COM ACIDENTE VASCULAR CEREBRAL EM HOSPITAL SECUNDÁRIO
}

\author{
MÁRCIA RADANOVIC*
}

\begin{abstract}
RESUMO - Objetivo: Descrever o perfil de atendimento de pacientes com acidente vascular cerebral (AVC) realizado no Hospital Universitário da Universidade de São Paulo (HU-USP), pela equipe de Clínica Médica do Setor de Emergência, como padrão de hospital secundário. Método: Análise retrospectiva de 228 prontuários de pacientes com doença cerebrovascular, no período de 1989 a 1993. Resultados: Encontramos a seguinte distribuição para os subtipos: $53 \%$ AVC isquêmico, $26 \%$ AVC hemorrágico, $2 \%$ associação de ambos e $19 \%$ não classificados. Incidência dos fatores de risco: hipertensão arterial sistêmica, 67,5\%; cardiopatias, 24,6\%, sendo mais frequente a fibrilação auricular crônica; diabetes, 20,2\%. Entre as complicações, coma foi constatado em 22,4\% dos casos; insuficiência respiratória, em 15\%; infecção, em 29\%. A taxa de óbito foi 14,5\%. A média de permanência no hospital foi 4,11 dias. Conclusões: Os aspectos epidemiológicos e complicações obtidos reproduziram o padrão descrito na literatura. Aspectos negativos observados foram a insuficiência de leitos e a incidência elevada de infecções pulmonares. A taxa de mortalidade foi comparável às mais baixas da literatura mundial.
\end{abstract}

PALAVRAS-CHAVE: doença cerebrovascular, epidemiologia, complicações.

\section{Characteristics of stroke assistance in a secondary hospital}

ABSTRACT - Objective: To describe the assistance profile of stroke patients accomplished in Hospital Universitário of University of São Paulo (HU-USP), by the Internal Medicine team of the Emergency Room, as being a standard of non-tertiary hospital. Method: Retrospective analysis of 228 patients with cerebrovascular disease, assisted from 1989 to 1993. Results: We found the following subgroup distribution: $53 \%$ of patients had ischemic strokes, $26 \%$, had hemorrhagic strokes, $2 \%$ had association from both and $19 \%$ were not classified. Among risk factors, we found an incidence of $67,5 \%$ for arterial hypertension; $24.6 \%$ for cardiac disease (chronic atrial fibrillation being the most frequent); diabetes occurred in $20.2 \%$ of cases. Among complications, coma was present in $22.4 \%$ of cases; respiratory insufficiency occurred in $15 \%$; infection, in $29 \%$. Death rate was $14.5 \%$. Average permanence in hospital was 4.11 days. Conclusions: The epidemiological aspects and complications reproduced the data described in the literature. Some negative aspects were inadequacy of accommodations and the great incidence of pulmonary infections. However, the mortality rate was comparable to the lowest found in literature.

KEY WORDS: cerebrovascular disease, epidemiology, complications.

A doença cerebrovascular (DCV) é causa importante de morbidade e mortalidade em nosso meio. Segundo registros dos Indicadores e Dados Básicos de 1997 do Ministério da Saúde, a mortalidade proporcional devida a doenças do aparelho circulatório é de 32,3\%, liderando as causas de óbito no Brasil. Dentro deste grupo, a DCV ocupa o primeiro lugar, sendo responsável por cerca de 1/3 das mortes, ultrapassando a doença isquêmica coronariana. A taxa específica de mortalidade

* Médica Pesquisadora de Departamento de Neurologia do Hospital das Clínicas da Faculdade de Medicina da Universidade de São Paulo (HC-FMUSP). Estudo realizado pela autora enquanto Médica Assistente Neurologista da Divisão de Clínica Médica do HU-USP. Aceite: 26-outubro-1999.

Dra. Márcia Radanovic - Rua Cristiano Viana 163/92 - 05411-000 São Paulo SP - Brasil. E-mail: mgomes@tecway.com.br 


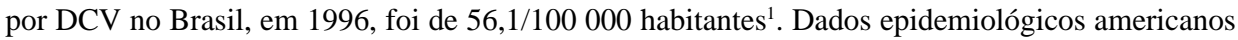
estimam que a DCV seja responsável por pelo menos $50 \%$ dos casos neurológicos atendidos num hospital geral ${ }^{2}$.

O termo acidente vascular cerebral (AVC) é usado para designar o déficit neurológico (transitório ou definitivo) em uma área cerebral secundário a lesão vascular, e representa um grupo de doenças com manifestações clínicas semelhantes, mas que possuem etiologias diversas: AVC hemorrágico (AVCh) compreende a hemorragia subaracnóide (HSA), em geral decorrente da ruptura de aneurismas saculares congênitos localizados nas artérias do polígono de Willis e a hemorragia intraparenquimatosa (HIP), cujo mecanismo causal básico é a degeneração hialina de artérias intraparenquimatosas cerebrais, tendo como principal doença associada a hipertensão arterial sistêmica (HAS); AVC isquêmico (AVCi) descreve o déficit neurológico resultante da insuficiência de suprimento sanguíneo cerebral, podendo ser temporário (episódio isquêmico transitório, EIT) ou permanente, e tendo como principais fatores de risco a HAS, as cardiopatias e o diabetes mellitus (DM). Outras etiologias podem estar associadas ao AVC, tais como coagulopatias, tumores, arterites inflamatórias e infecciosas. Este conjunto de doenças representa grande ônus em termos sócioeconômicos, pela alta incidência e prevalência de quadros sequelares. A importância da DCV para o Sistema de Saúde no Brasil pode ser estimada pelo fato de representar 8,2 \% das internações e $19 \%$ dos custos hospitalares do Instituto Nacional de Assistência Médica da Previdência Social (INAMPS) ${ }^{3}$.

A maior parte do atendimento de pacientes com AVC no Brasil é realizada em hospitais secundários. Hospitais deste porte muitas vezes não dispõem de uma infra-estrutura adequada para o atendimento completo deste tipo de doente. Nosso estudo descreve o perfil de atendimento realizado no Hospital Universitário da Universidade de São Paulo (HU-USP), pela equipe de Clínica Médica do Setor de Emergência. Trata-se de estudo retrospectivo, enfocando aspectos epidemiológicos, clínicos e evolutivos de interesse para melhor compreensão de como pode ser possível a otimização dos recursos disponíveis nos hospitais de menor complexidade. O HU-USP é um hospital-escola onde realizam estágio alunos de terceiro, quinto e sexto anos do curso de Medicina da Faculdade de Medicina da USP, bem como alunos da Escola de Enfermagem da USP, entre outros profissionais da saúde. Por este motivo, este estudo serviu de modelo para a implantação de melhorias no atendimento do paciente com AVC, tendo sido efetuado na forma de análise retrospectiva quando o hospital incorporou médico neurologista em sua equipe de Clínica Médica.

\section{MÉTODO}

O estudo é baseado na análise retrospectiva de 228 prontuários de pacientes classificados com o diagnóstico de DCV (CID 430-438), atendidos no Setor de Emergência do HU-USP pela equipe de Clínica Médica no período de 1989 a 1993. Foram considerados apenas os casos em que o motivo principal da procura do atendimento de emergência foi o evento cerebrovascular. $O$ grupo estudado era composto de uma população de adultos (acima de 25 anos), sendo 124 homens (54\%) e 104 mulheres (46\%).

Nosso levantamento procurou caracterizar alguns aspectos referentes a: apresentação clínica na sala de admissão (nível de consciência, infecção domiciliar associada); doenças associadas ao quadro (HAS, DM, cardiopatias); perfil de distribuição dos diferentes subtipos de AVC na composição do total de atendimentos; evolução clínica e complicações (rebaixamento do nível de consciência, insuficiência respiratória, aquisição de infecção hospitalar); tipo de cuidado requerido pelo paciente (necessidade de avaliação por neurologista, remoção para realização de exames subsidiários); tempo de permanência no hospital (incluindo local de acomodação).

O estudo estatístico foi realizado com uso do teste do qui quadrado $\left(\chi^{2}\right)$ para verificar possíveis associações entre variáveis, sendo aceito $\alpha=0,01$ para interpretação dos resultados.

\section{RESULTADOS}

Os dados recolhidos demonstram a frequência de distribuição de cada subtipo de AVC ocorrendo da forma exposta na Figura 1. Em grande porcentagem de casos (62\%), não houve definição do diagnóstico. O perfil dos resultados da TC de crânio é mostrado na Figura 2, notando-se que 19\% 


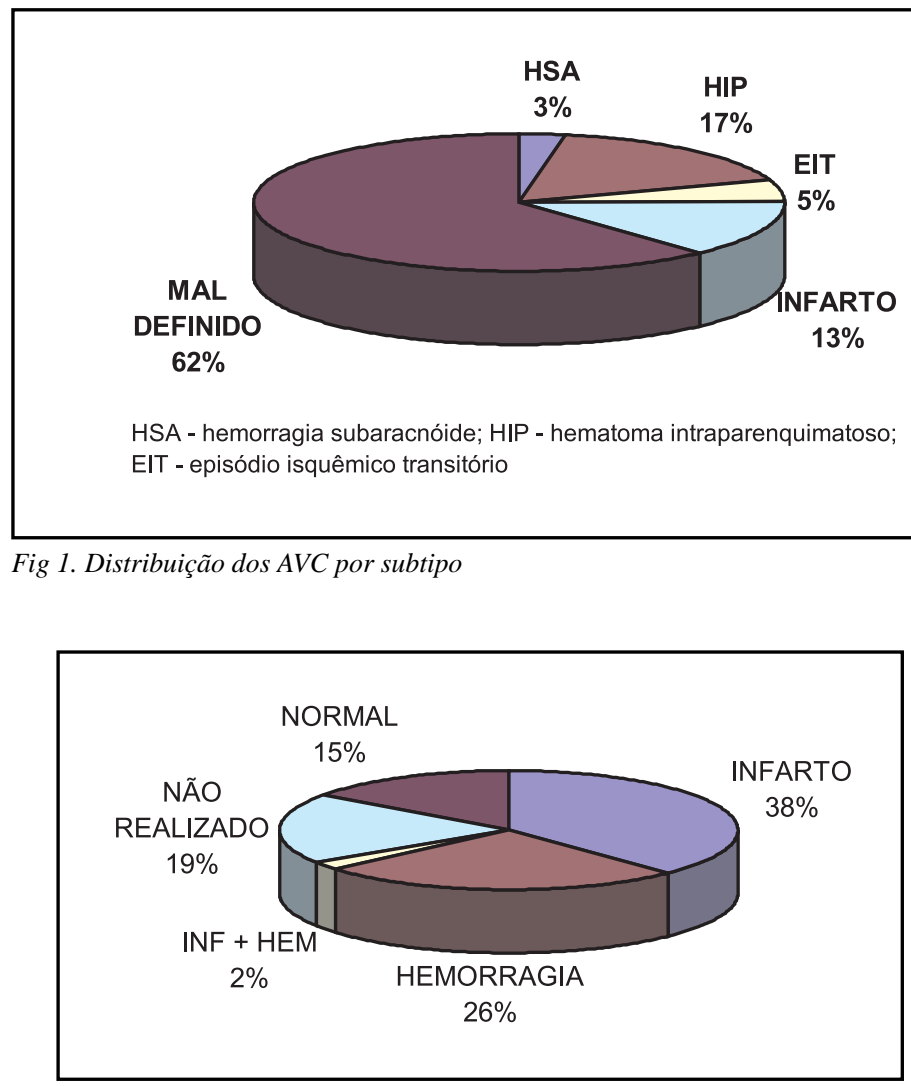

Fig 2. Diagnóstico do AVC por TC de crânio

dos pacientes não realizaram o exame, recebendo portanto um diagnóstico baseado em critérios clínicos.

Para efeito da análise de distribuição dos eventos de acordo com o subtipo de AVC, utilizamos o diagnóstico tomográfico. Desta forma, foram excluídos os pacientes que não realizaram a TC de crânio e aqueles em que o diagnóstico incluía infarto e hemorragia associados, pela impossibilidade de distinguir, com os dados de prontuário disponíveis, se o quadro primário apresentado pelo paciente havia sido hemorrágico (por exemplo, hemorragia subaracnóide com isquemia secundária a vasoespasmo) ou isquêmico (AVCi com transformação hemorrágica). Desta forma, o total de casos cuja análise aparece discriminada foi 180 pacientes (53\% apresentando AVCi e 26\% apresentando AVCh).

No tocante às doenças associadas ao AVC recente, encontramos a distribuição exposta na Tabela 1. Dentre as cardiopatias, a mais encontrada foi a fibrilação atrial (FA) crônica, documentada em 11,9\% dos pacientes (com frequência relativa de 22,1\% nos pacientes com AVCi e 3,4\% nos casos de $\mathrm{AVCh}$ ). A presença de área inativa no eletrocardiograma (ECG), indicando doença isquêmica coronariana foi constatada em $8,3 \%$ do total de pacientes, com frequência relativa de $10,6 \%$ nos que sofreram AVCi e de 6,1\% nos que apresentaram quadro hemorrágico. Com relação à investigação etiológica do quadro, notamos que em 30,7\% dos casos, o ECG não foi realizado durante todo o período de internação do paciente e em $21,1 \%$ dos casos não há referência no prontuário sobre o paciente ser ou não diabético. 
Tabela 1. Frequência da associação das principais doenças que atuam como fator de risco para AVC (porcentagem de ocorrência).

\begin{tabular}{cccc}
\hline Tipo & & Doença associada & \\
AVC & cardiopatia & DM & HAS \\
\hline Total & 24,6 & 20,2 & 67,5 \\
AVCI & 41,9 & 23,3 & 60,5 \\
AVCH & 1,7 & 8,5 & 83,1 \\
\hline
\end{tabular}

DM, diabetes mellitus; HAS, hipertensão arterial sistêmica.

Em 71,9\% dos casos, os pacientes não tinham antecedente de AVC; $21,5 \%$ já haviam apresentado um episódio; $3,5 \%$ dos pacientes tinham 2 AVCs pregressos; $0,4 \%$ referiam 3 episódios anteriores e $1,7 \%$ já tinha apresentado mais de 3 episódios. Ainda com relação ao evento em questão, 89,5\% dos pacientes negaram a ocorrência de sintomas compatíveis com EIT antecedendo o quadro (83, 7\% dos AVCi e 98,3\% dos AVCh). Obtivemos referência positiva em 8,3\% dos casos (12,8\% dos pacientes com AVCi e 1,7\% naqueles com AVCh). No restante dos casos, o quesito não foi registrado.

Os dados mais relevantes referentes a aspectos da evolução e complicações clínicas estão expostos na Tabela 2. Alguns detalhes sobre a apresentação das infecções encontradas encontramse na Tabela 3 e Figura 3. As infecções pulmonares lideraram o número de ocorrências (79\% do total), tanto entre as adquiridas na comunidade quanto nas hospitalares. Dentre os quadros extrapulmonares, o sítio de infecção mais frequente foi o trato urinário.

Com relação ao local de permanência dos pacientes, constatamos que 54,5\% dos pacientes permaneceram no hospital por mais de 72 horas, sendo que isto ocorreu com maior frequência no subgrupo de pacientes com AVCh (72,9\%, contra 65,1\% nos casos de AVCi); 22,1\% dos casos puderam receber alta hospitalar em menos de 72 horas (sendo 25,6\% dos AVCi e apenas 5,1\% dos AVCh). Um total de 17,3\% dos pacientes foi transferido para outro serviço por falta de disponibilidade de leitos para acomodação, para tratamento mais especializado (intervenção neurocirúrgica, por exemplo) ou porque o paciente tinha direito a alguma espécie de atendimento com maior grau de conforto, como convênio hospitalar. Dos pacientes que não foram transferidos, apenas $46,5 \%$ puderam ser internados na enfermaria ou UTI, sendo que o restante $(53,5 \%)$ recebeu todo o atendimento no próprio Setor de Emergência (sala de admissão ou retaguarda). A média de permanência dos pacientes no hospital foi 4,11 dias, sendo 4,25 dias para o subgrupo AVCh e 3,87 para o AVCi .

Tabela 2. Evolução e complicações clínicas dos pacientes com AVC (porcentagem de ocorrência).

\begin{tabular}{lcccc}
\hline Tipo de & \multicolumn{2}{c}{ Evolução / complicação clínica } \\
AVC & Coma & Insuf. resp. & Infecção & Óbito \\
\hline Total & 22,4 & 14,0 & 29,0 & 14,5 \\
AVCI & 24,4 & 16,3 & 46,6 & 16,3 \\
AVCH & 28,0 & 15,3 & 30,6 & 18,7 \\
\hline
\end{tabular}




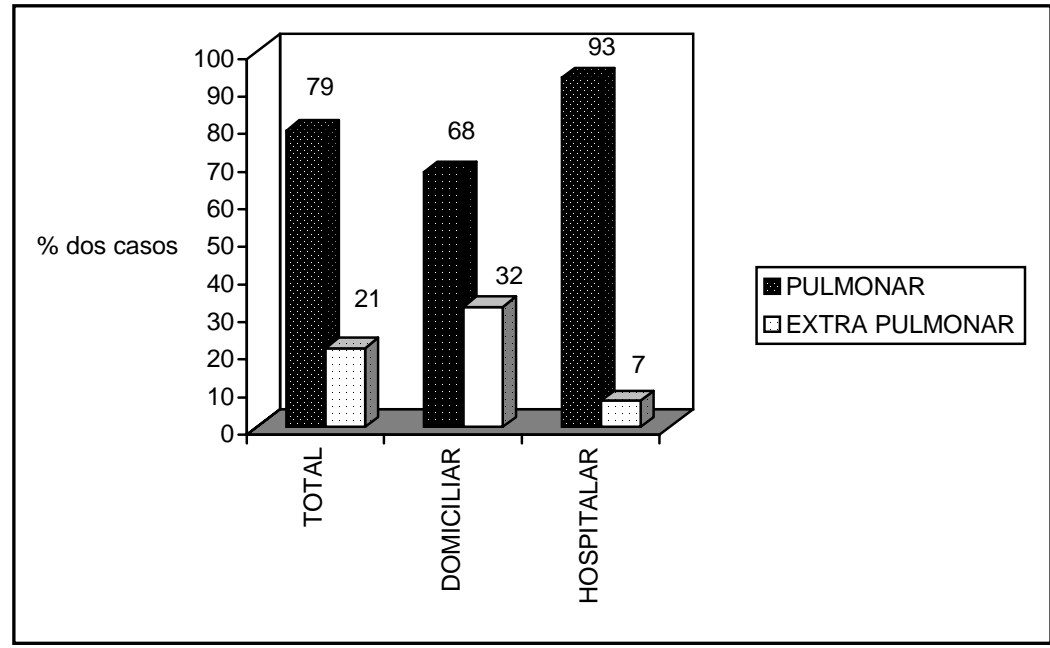

Fig 3. Distribuição relativa do sítio de infecção nos pacientes com AVC.

Tabela 3. Distribuição da porcentagem de infecções de acordo com o local de aquisição.

\begin{tabular}{lccc}
\hline Local de aquisição & \% Total de pacientes & \% AVCI & $\%$ AVCH \\
\hline domiciliar & 16,3 & 25,6 & 13,6 \\
hospitalar & 12,7 & 21,0 & 17,0 \\
Total & 29,0 & 46,6 & 30,6 \\
\hline
\end{tabular}

Em 50,4\% dos casos, a equipe clínica sentiu necessidade de encaminhar o paciente a um serviço terciário para realizar avaliação neurológica. Os principais motivos para esta solicitação foram realização ou interpretação de TC de crânio, questões sobre indicação de anticoagulação e avaliação por equipe de Neurocirurgia.

\section{DISCUSSÃO}

Nas últimas décadas, uma enorme quantidade de recursos tem sido investida em pesquisa, no mundo todo, na tentativa de reduzir a morbidade e mortalidade dos AVC. Várias modalidades terapêuticas têm sido preconizadas, todas objetivando minimizar o grau de lesão neuronal que ocorre após uma oclusão ou sangramento arterial. Assistimos ao desenvolvimento das intervenções objetivando a otimização do fluxo sanguíneo (farmacológicas e cirúrgicas), sobre o metabolismo neuronal (drogas neuroprotetoras, uso de agentes anestésicos), controle agressivo da hipertensão intracraniana, aperfeiçoamento das técnicas neurocirúrgicas, permitindo maior segurança na sua indicação e execução. Além disso, um intenso esforço tem sido despendido na tentativa de controlar os fatores de risco e na profilaxia de novos eventos, também através da terapêutica medicamentosa ou intervenção cirúrgica precoce (endarterectomias) nos pacientes de alto risco. No entanto, vivemos uma realidade em que a maior parte dos pacientes com AVC recebe o primeiro atendimento em centros onde não existem especialistas ou Serviços de Neurologia. Este atendimento vai ser realizado, em grande parte, pelo clínico geral. Os resultados do nosso levantamento mostram que, em relação ao diagnóstico, a equipe médica apresentou alguma dificuldade em manusear adequadamente os conceitos necessários para categorizar os AVC: em $62 \%$ dos casos, o diagnóstico final do paciente foi "Doença cerebrovascular mal definida", mesmo levando-se em consideração que grande parte 
dos pacientes nesta categoria havia realizado TC de crânio. Outro dado que chama atenção em relação ao procedimento diagnóstico é o fato de que $19 \%$ dos pacientes não realizaram TC de crânio, sendo o diagnóstico atribuído com base apenas em critérios clínicos. Tal fato certamente tem impacto negativo sobre o tratamento do AVC na fase aguda, bem como sobre a possibilidade de orientar adequadamente o paciente do ponto de vista da profilaxia de novos eventos, dada a dificuldade de se estabelecer qual o tipo e etiologia do AVC sem exame de neuroimagem. Num grupo de 1059 pacientes com suspeita de AVC, Weir et al. ${ }^{4}$, demonstraram a ineficácia de escores baseados apenas em dados clínicos para diferenciar quadros isquêmicos e hemorrágicos, mesmo quando as variáveis clínicas analisadas incluíram dados epidemiológicos, fatores de risco e histórico de doença cardiovascular pregressa extremamente detalhados, além do quadro clínico apresentado pelo paciente.

A hipertensão arterial é fator de risco preditivo poderoso para o AVC. Sua ocorrência está estimada em torno de $70 \%$ de todos os quadros vasculares cerebrais ${ }^{5}$. Num estudo de 1985 , englobando 1088 registros de pacientes, Lessa ${ }^{6}$ encontrou uma incidência de $80 \%$ de hipertensos. Tal achado se confirmou em nossa amostra, na qual constatamos frequência de 67,5\% para todos os AVC (Tabela 1). Com relação ao subtipo, encontramos uma associação mais significativa com os quadros hemorrágicos $(\mathrm{p}<0,01)$, dado também reportado no estudo citado acima.

Doenças cardíacas constituem o segundo mais importante fator de risco para AVC, especialmente para os quadros aterotrombóticos e embólicos. A prevalência de cardiopatias em nossa amostra foi 24,6\% (Tabela 1), cerca de 25 vezes mais frequente na população que apresentou AVCi do que no grupo que sofreu AVCh; FA crônica foi a anormalidade mais encontrada, presente em 22,1\% dos quadros isquêmicos. Tal distribuição repercute na morbi-mortalidade da doença, uma vez que os quadros embólicos apresentam risco de complicação potencial mais elevado, seja pela maior extensão da área infartada, pela possibilidade de ocorrência de transformação hemorrágica ou pela necessidade de anticoagulação. Observamos neste estudo que o tratamento destes pacientes sem a intervenção de um especialista representou dificuldade adicional para a equipe clínica, e motivou grande número de transferências para serviço terciário a fim de se obter parecer do neurologista quanto à adoção ou não da anticoagulação. Da mesma forma como observado em relação ao diabetes, quase $1 / 3$ dos pacientes não realizou ECG durante o primeiro atendimento. Isto não permite uma análise mais detalhada da correlação entre evidência de doença isquêmica coronariana e AVC.

Diabetes mellitus, reconhecidamente um fator de risco independente para a DCV por acelerar o processo de aterosclerose ${ }^{7}$, foi encontrado em 20,2 \% dos pacientes (Tabela 1). Também aqui, a maior ocorrência entre os indivíduos com $\mathrm{AVCi}$, quando comparado ao grupo com AVCh, foi estatisticamente significante ( $\mathrm{p}<0,01)$. A impossibilidade de se obter este dado em mais de $1 / 5$ dos prontuários tem dois significados igualmente relevantes: primeiro, o dado não foi colhido durante a realização da história clínica; segundo, durante o período de tratamento da fase aguda do AVC não foi realizado exame de glicemia. Tendo em vista a importância do controle dos fatores de risco para a profilaxia de novos eventos e do controle metabólico para melhor evolução do quadro instalado, este aspecto do atendimento não pode ser neglicenciado.

O índice de piora dos pacientes com AVC nos primeiros sete dias após o icto varia em torno de $30 \%$ na literatura ${ }^{8,9}$. Destes, $70 \%$ pioram por causas neurológicas (edema cerebral, progressão da área infartada, transformação hemorrágica, vasoespasmo, crises convulsivas, hidrocefalia aguda e ressangramento) e $30 \%$ por causas sistêmicas ${ }^{8}$. A frequência de pacientes em coma observada $22,4 \%$ (Tabela 2), aproxima-se do resultado de estudo epidemiológico realizado em Salvador, Bahia, em que este índice foi de $34,9 \%^{10}$. Não houve diferença estatisticamente relevante entre os dois subgrupos de AVC em nossa casuística.

Distúrbios respiratórios são causa frequente de complicação no AVC, perdendo apenas para os distúrbios cardíacos entre as causas não-neurológicas de piora após um quadro vascular cerebral ${ }^{11}$. Broncopneumonia, pneumonia lobar, embolia pulmonar e acúmulo de secreção tráqueo-brônquica são os eventos mais frequentes, todos podendo levar a insuficiência respiratória. A restrição ao leito, pouca 
movimentação, limitada expansão da caixa torácica, disfagia, uso de sonda naso-gástrica e instituição de ventilação mecânica são os fatores responsáveis pela alta incidência de infecções respiratórias nestes pacientes. A hipóxia e hipercapnia decorrentes deste estado, bem como o componente tóxico-infeccioso do processo agravam a lesão cerebral. Dados do estudo RANTTAS (Randomized Trial of Tirilazad in Acute Stroke $)^{12}$ mostram incidência de 5\% de infecções pulmonares numa série de 279 pacientes com quadros cerebrovasculares isquêmicos. Arboix et al. ${ }^{13}$, em Barcelona, descreveram pneumonia como complicação em $14 \%$ de 1356 casos de AVC. A incidência de pneumonia encontrada em nossa população superou grandemente os índices habitualmente reportados na literatura, tanto de infecções domiciliares como nas adquiridas no hospital (Tabelas 2 e 3 ). A proporção relativa de infecção adquirida em ambiente domiciliar e hospitalar é semelhante, tanto no total de pacientes como para os subgrupos (Tabela 3). Acreditamos que o alto índice de pacientes que já chegam ao hospital infectados possa ser explicado em termos da demora em ser removido ao serviço de emergência, mesmo em casos em que ocorre alguma alteração do nível de consciência, como sonolência, o que predispõe a aspiração. Em nossa cultura existe o hábito arraigado de "alimentar o doente para que ele melhore", mesmo quando o indivíduo não se encontra em condições de ingerir alimentos, contribuindo para o alto número de pneumonias aspirativas. Em contrapartida, o elevado número de infecções intra-hospitalares deriva em grande parte da precária disponibilidade de atendimento fisioterápico mesmo nos pacientes que se encontram em ventilação mecânica nas unidades de terapia intensiva (UTI).

A taxa de mortalidade para os AVC varia, na literatura recente, entre 14 e $26 \% \%^{12-16}$. Nosso índice de óbito (14,5\% do total de 189 pacientes não transferidos) encontra-se no limite inferior desta variação. A diferença entre os dois subgrupos não foi estatisticamente significante, apesar de haver uma crença generalizada entre a população médica de que as hemorragias apresentam quadro clínico mais grave e maior probabilidade de êxito letal. Os pacientes com quadros hemorrágicos, entretanto, tiveram tempo de internação mais prolongado do que os acometidos por eventos isquêmicos ( $\mathrm{p}<0,01$ ); ainda assim, o tempo médio de internação foi baixo. Constatamos ainda que mais de $50 \%$ dos pacientes não puderam ser adequadamente acomodados em um leito de enfermaria, recebendo todo o atendimento, até a alta, na sala de admissão ou retaguarda do Setor de Pronto Atendimento. Esses números refletem uma tendência generalizada nos serviços de emergência em nosso país, que é a de liberar o paciente precocemente, mesmo diante de uma patologia com potencial para complicações, devido à superlotação e inadequação entre a demanda e disponibilidade de leitos, problemas crônicos em nossos hospitais públicos.

\section{CONCLUSÕES}

$\mathrm{O}$ atendimento adequado ao paciente com AVC ainda constitui um desafio, pelo alto potencial de morbidade e mortalidade associados a este diagnóstico. O cuidado ideal deste paciente é caro, pois demanda a realização de exames subsidiários de alto custo para confirmação do diagnóstico, etiologia e planejamento terapêutico (TC de crânio, arteriografia cerebral, ressonância magnética de encéfalo), pode requerer internação em UTI ou intervenção neurocirúrgica e necessita de equipe de reabilitação desde as fases mais precoces. Os principais problemas detectados com relação a este atendimento, em nosso estudo, foram: necessidade de remoção do paciente a um serviço terciário para avaliação neurológica em cerca de metade dos casos, aumentando os riscos e o custo do tratamento; insuficiência de leitos para internação; a incidência de infecções pulmonares superou em muito a descrita como aceitável atualmente em centros mais avançados, o que pode ser atribuído em grande parte às condições precárias de acomodação e à indisponibilidade de tratamento fisioterápico profilático.

No tocante aos aspectos epidemiológicos, observamos coexistência de fatores de risco para os subtipos de AVC compatível com os dados da literatura. As complicações também seguiram o padrão classicamente descrito e, apesar dos fatores desfavoráveis já descritos, a taxa de mortalidade encontrada nesta casuística foi comparável às mais baixas referidas na literatura mundial recente.

Assim, parece-nos que o perfil aqui apresentado reflete dificuldades comuns ao serviço público não-terciário, sendo representativo dos principais problemas cuja solução pode contribuir, ao lado 
do investimento no adequado controle dos fatores de risco e na profilaxia de novos eventos em paciente suscetíveis, para atenuar o impacto da DCV sobre o indivíduo e a sociedade.

Agradecimentos - A autora agradece ao Dr. Paulo Andrade Lotufo, pelo auxílio na organização dos dados coletados.

\section{REFERÊNCIAS}

1. Ministério da Saúde - Sistema de Informações sobre Mortalidade. DATASUS - TABNET. Indicadores e Dados Básicos Brasil 97.www.datasus.com.br, 1999.

2. Adams RD, Victor M, Ropper AH. Cerebrovascular diseases. In: Adams RD, Victor M, Ropper AH (eds) Principles of neurology. 6Ed. New York: McGraw-Hill, 1997: 777-873.

3. Gomes MM. Doenças do cérebro: prioridade de política de saúde pública no Brasil? Rev Bras Neurol 1992;28:11-16..

4. Weir CJ, Murray GD, Adams FG, Muir KW, Grosset DG, Lees, KR. Poor accuracy of stroke scoring systems for differential clinical diagnosis of intracranial haemorrhage and infarction. Lancet 1994;334:999-1002.

5. Dunbabin DW, Sandercock PAG. Preventing stroke by the modification of risk factors. Stroke 1990;21 (Suppl 4):36-39.

6. Lessa I. Hipertensão arterial e acidente vascular encefálico em Salvador, Bahia. Rev Assoc Méd Bras 1985;31:232-235.

7. Weinberger J, Biscarra V, Weisberg MK, Jacobson JH. Factors contributing to stroke in patients with atherosclerotic disease of great vessels: the role of diabetes. Stroke 1983;14:709-712.

8. Hachinski W, Norris JW. The acute stroke. Philadelphia: F. A. Davis Company, 1985: 123-140.

9. Britton M, Roden A. Progression of stroke after arrival at hospital. Stroke 1985;16: 629-632.

10. Lessa I. Epidemiologia dos acidentes vasculares encefálicos na cidade de Salvador: aspectos clínicos. Arq Neuropsiquiatr 1985;43:133-139.

11. Gagliardi RJ Acidente vascular cerebral: fatores de piora dos doentes nos primeiros dias. Rev Bras Clin Terap 1988;17:155-159.

12. Johnston KC, Li JY, Lyden PD, et al. Medical and neurological complications of ischemic stroke experience from the RANTTAS trial: RANTTAS investigators. Stroke 1998;29:447-453.

13. Arboix A, Massons J, Oliveres M, Garcia L, Titus F. Mortalidad en la fase aguda de la enfermedad cerebrovascular: registro de La Alianza - Hospital Central de Barcelona. Med Clin (Barc) 1994;103:529-533.

14. Anderson CS, Jamrozik KD, Broadhurst RJ, Stewart-Wynne EG. Predicting survival for 1 year among different subtypes of stroke: results from the Perth Community Stroke Study. Stroke 1994;25:1935-1944.

15. Akai T, Takahata T, Sugita K, Sonobe M, Takahashi S. The outcome of cerebrovascular disease in the elderly. No To Shinkei 1997;49:337-341.

16. Cabral NL, Longo A, Moro CHC, Amaral CH, Kiss, HC. Epidemiologia dos acidentes cerebrovasculares em Joinville, Brasil: estudo institucional. Arq Neuropsiquiatr 1997;55:357-363. 